\title{
The Wrap-Up: A Unique Forum To Support Pediatric Residents When Faced with the Death of a Child
}

\author{
Scot T. Bateman, M.D., ${ }^{1}$ Rebecca Dixon, M.D., ${ }^{2}$ and Maria Trozzi, M. Ed. ${ }^{3}$
}

\begin{abstract}
Objective: The project intended to describe the format of the Wrap-up, a unique multidisciplinary guided debriefing following a child's death. Specific feedback from pediatric residents was sought to assess the model. Methods: The Wrap-ups were timely (within 48 hours of a death), consistent (conducted after each pediatric intensive care unit (PICU) death), multidisciplinary (all care providers were invited), and specifically conducted by someone trained in postdeath facilitation. The role of the conductor was focused on being inclusive, navigating the discussion, diffusing areas of conflict or angst, and managing the tone of the meeting.

Resident feedback was obtained by a one-time (May 2010) anonymous internet-based survey, with both openended free-text questions and five-point Likert scale queries. Surveyed were all residents rotating though the PICU between 2007 and 2010. Open-ended free-text responses were analyzed using content analysis methods by combining recurrent themes and organizing by main components of the Wrap-up. Quantitative responses, via a five-point Likert scale, were averaged.

Results: Between 2007 and 2010, there were 36 PICU deaths. The average age was nine years old. All deaths had an accompanying conductor-led Wrap-up occurring, on average, two days after the death.

Sixty percent $(27 / 45)$ of pediatric residents completed the survey. Their qualitative responses showed that the key components (timely, multidisciplinary, and specifically conducted) of the Wrap-ups were valuable. Quantitatively, they agreed or strongly agreed that the consistent Wrap-ups improved end-of-life care, teamwork, stress surrounding the death, and the ability to care for others.

Conclusion: The Wrap-up, a unique forum for debriefing after a pediatric death, was well-received by residents and assisted them with processing, understanding, and resolving their experience regarding the pediatric death. The Wrap-up was a valuable addition to residents' experience and education in pediatric critical care medicine and can be replicated in other institutions.
\end{abstract}

\section{Introduction}

$\mathbf{P}$ EDIATRIC DEATHS IN the hospital, though relatively rare ( $1 \%-2 \%$ of total admissions), can induce a significant emotional toll on health care providers. ${ }^{1-4}$ This is particularly relevant in the pediatric intensive care unit (PICU), where two-thirds of hospitalized children die, and two-thirds of those are the result of withdrawal of support. ${ }^{2,3}$ Pediatric residents, who may be experiencing a child's death for the first time in the PICU, are likely to be unprepared for this burden. ${ }^{1,5-7}$ Consistent efforts to provide support for residents and staff after a pediatric death in the PICU could help with this burden.
National guidelines on end-of-life care in intensive care units strongly suggest staff support mechanisms should be offered, but no specific recommendations are available. ${ }^{8} \mathrm{Be}-$ reavement rounds or debriefings have been reported in the literature as a major form of coping for pediatric providers. However, reports indication that the incidence of any form of debriefing after a death hovers around $30 \%$ of the deaths. ${ }^{9-11}$ Factors that influenced when a debriefing was conducted were deaths that had a particular emotional toll, such as the death of a previously healthy child, or ones that involved specific medical issues. ${ }^{10,11}$ Although some deaths induce more emotional toll, each PICU death could be stressful to the care team and therefore warrants discussion and a chance to learn from others.

\footnotetext{
${ }^{1}$ University of Massachusetts Medical School, Worcester, Massachusetts.

${ }^{2}$ Primary Children's Medical Center, University of Utah, Salt Lake City, Utah.

${ }^{3}$ Boston University School of Medicine, Boston, Massachusetts.

Accepted July 31, 2012.
} 
The Wrap-up model was originated by Maria Trozzi, M.Ed., director of the Good Grief Program, at Boston University School of Medicine. She sought to create a forum that could provide consistent support and be perceived by residents as helpful, and used a technique with which they were already comfortable. It started with creating an expectation that for every pediatric death, a Wrap-up would be held. Rounds each day in the PICU are multidisciplinary, as is the care that is delivered; so, the entire care team was involved when a child died. The Wrap-up sought to create a similar and familiar approach to postdeath rounds by having all disciplines involved. In addition, attending-level leadership, as at all other significant teaching forums during residency, provided legitimacy. As this type of postdeath leadership was likely to be unfamiliar, even to very experienced senior staff, Trozzi created a 30-hour training program for staff physicians. This training focused on the leader's capacity to develop a prescribed technique for gently leading clinicians through the events to create a sequential narrative of the death experience, be sensitive to listening to all participants, redirect comments that may be judgmental, and differentiate content that is affective and cognitive. These concepts were derived from her book focused on death of children. ${ }^{12}$ This training aimed to help the leaders to be "conductors" of the meeting rather than facilitators. "Conduction" was chosen specifically to imply that, with good leadership, the meeting could be predictable and sequential in its unfolding of information regarding the patient's care, and carry an additional bonus of a sense of competency, adequacy, relatable success, and teamwork.

The PICU at Umass Memorial Medical Center has been able to maintain postdeath Wrap-ups since 2006, when an attending (STB) joined the staff who had completed the training program for conductors. This article was written to present a description of this unique forum for staff and resident support after a pediatric death in the PICU. The Wrap-ups were timely, multidisciplinary, and specifically conducted to enhance communication. We additionally aimed to highlight the benefits of this model by presenting survey results from pediatric residents on their experiences with them.

\section{Methods}

Each Wrap-up followed a similar format, which is summarized in the appendix. Immediately after a death in the PICU, our division administrator was notified, and a meeting was coordinated within 48 hours. All multidisciplinary care providers who had contact with the child were invited, including attendings, residents, medical students, nursing (night and day staff), aides, respiratory therapists, primary care providers, consultants, chaplains, child life representatives, social workers, and unit secretaries. Every attempt was made to choose a time that would have the most attendance. One hour was allotted for the meeting.

Each Wrap-up was led by a trained attending physician (the "conductor") (lead author STB) who participated in the 30-hour training for conductors. The conductor introduced the "norms" or "rules" for participation. This included stressing that participation in the discussion was entirely voluntary, that all have an equal voice and that all topics discussed would be confidential among participants. The conductor may or may not have had direct knowledge of the case being discussed. The conductor asked for a general medical sum- mary from a volunteer. The conductor then guided the discussion with open-ended questions, often asking anyone who wished to speak to assist in telling the "narrative." Attempts were made to avoid focusing primarily on medical issues (i.e., morbidity and mortality presentation/analysis) and focusing towards the humanistic issues at the end of life. For example, "Who was there when the child died?" or "What were the parents doing at this point?" The conductor also encouraged participation as the chronology of care unfolded, particularly given the group's hierarchy. The conductor had to improvise and attempted to identify areas of conflict or angst or sadness from those participating. When conflict was present, the conductor worked to develop consensus and/or reconciliation. In many cases, an appreciation of what was done well by the staff at the end of life was often a focus point for discussion.

The conductor carefully attempted to maintain the discussion in the "cognitive domain," or thoughts expressed, rather than the more obvious "affective domain," or feelings expressed. This often was difficult to achieve when emotional content was intense, but could be done by gently acknowledging emotions and redirecting towards concrete aspects of the dying experience. This was done to promote an "emotionally safe" environment for the participants and was critical for the success of the Wrap-up. The overall effect allowed for emotional content to be expressed without it becoming dominant, incapacitating, or draining.

The pediatric resident feedback was sought via a onetime internet-based survey (www.surveymonkey.com/s/ 6MCXDYD) sent in May 2010 from a fellow resident (RDD) to all residents who rotated through the PICU from 2007 to 2010. Since the lead author (STB) was the conductor for the Wrapups, feedback was sought independently of him to avoid any conflicts of interest in the feedback. Both categorical and internal medicine/pediatric residents (12 per year) rotate through the PICU for a minimum of three months during their residency training.

The survey had both open-ended questions with free-text responses and specific questions with a five-point Likert scale response (1, strongly disagree to 5 , strongly agree). The openended text questions (e.g., 'What was the most valuable aspect of the Wrap-Up?') were categorized using content analysis techniques. All free-text responses were combined and then categorized by theme. Verbatim responses were coded independently by two investigators with very high intercoder agreement (95\%). The predominant themes were then organized and reported as they related to the main components of the Wrap-up: (1) timeliness within 48 hours, (2) the multidisciplinary aspect, and (3) the role of the conductor. Quantitative responses (via the five-point Likert scale) to nine specific questions about potential benefits to resident education and coping mechanisms from the Wrap-ups were averaged and presented in graphical format.

\section{Results}

Between January 2007 and June 2010, in an 11-bed combined medical/surgical PICU, with approximately 700 annual admissions, there were 36 deaths for a mortality rate of $1.4 \%$.

The average age of those who died was nine years old and they are categorized in the Table 1.

Wrap-ups were completed for each death. The average time from death to the Wrap-up was two days. All Wrap-ups, 
Table 1. Deaths in PICU 1/2007-6/2010

\begin{tabular}{lr}
\hline & $N(\%)$ \\
\hline $\begin{array}{l}\text { Number } \\
\text { Average age: } 9.01 \text { years }\end{array}$ & $36(100)$ \\
Cause of death & \\
CNS disease & $6(16)$ \\
Multisystem organ failure & $6(16)$ \\
Anoxia & $3(8)$ \\
Trauma & $14(39)$ \\
Progression of underlying disease & $10(28)$ \\
Death circumstances & \\
Organ donation & $8(22)$ \\
Failed CPR & $5(14)$ \\
Withdrawal of support & $23(64)$ \\
\hline
\end{tabular}

CNS, central nervous system; CPR, cardiopulmonary resuscitation; PICU, pediatric intensive care unit.

but one, were conducted by a PICU staff attending with conducting training. The attendance ranged from five to 30 participants.

The anonymous survey was completed by 27 (60\%) of 45 residents who rotated through the PICU. All respondents had been to at least one Wrap-up. Fifteen (56\%) had been to 3-5 Wrap-ups, and $26 \%$ had been to $1-2$ Wrap-ups. Two had been to 5-10 Wrap-ups and three had been to $>10$. The number of Wrap-ups attended by the nonresponders is unknown.

All of the residents' qualitative responses regarding the three predetermined components (timeliness, multidisciplinary, specifically conducted) are listed below. Overall, their comments describe the strengths of the Wrap-up model, and what they gained from participating in this type of process.

\section{Timely}

The majority of comments confirmed why timeliness of the Wrap-up was important. They reported feeling that the effectiveness of the Wrap-up was enhanced by having it soon after a death (within two days) while the feelings and emotions were still "fresh." There was also a feeling of "importance" created by quickness of coordination of the Wrap-up that improved their desire to participate. This sense of importance helped the resident to "honor the stress" of a child's death.

"Things move fast in the hospital and emotions fade with time...especially for the residents who have to switch focus often."

"The meetings often help me process and acknowledge my stress and grief, and having them soon after the event makes them most helpful."

\section{Multidisciplinary}

The multidisciplinary nature of the Wrap-up provided important care team cohesiveness that enhanced resident processing of the death. There was an appreciation of "hearing from others on the team" so that the residents "didn't feel alone" and felt an improved sense of teamwork. In particular, there were repeated comments, from almost all responders, about the benefit of learning about the death from "multiple perspectives", particularly ones who have more experience (i.e., PICU nurses and attendings).
"I think the meetings foster a greater sense of teamwork. We see colleagues on a more personal level....Ultimately, I think this helps us as residents broaden our own scope as to how we see patients, families, and different situations.

"It is great to have everyone involved in the case because they may look at things differently or know things that you didn't that can help you cope better."

\section{Conducted}

A key aspect of the Wrap-up was the trained conductor who navigated the discussion. Respondents repeatedly commented that the conductor was effective at "setting the tone," "moving things along," "being nonjudgmental," and allowing everyone a "chance to speak." They felt that s/he was able to keep the discussion "under control", and making sure that it was "safe to share."

"They keep it simple and lead by example...."

"I think the conductor ties everything together and gives everyone a chance to speak and be heard. He/she also tries to hear from different people in the care team."

"Directs, focuses, and often prompts discussion."

\section{Impact on resident education}

The residents were overwhelmingly positive about their experiences at Wrap-ups (see Fig. 1). The vast majority of residents ( $>90 \%$ agreed, or strongly agreed) with all nine proposed statements of potential benefits.

Suggestions for improvement of Wrap-ups were also elicited. Most responses centered on ways to improve attendance. They stressed the importance of making sure that as many different staff involved in the care of the patient were able to come. There was never a time that everyone could make it, but some felt waiting a little longer to get the most people to attend would be helpful. They wanted the time to be officially freed up from other duties in order to attend.

\section{Discussion}

Although the death of a pediatric patient may be one of the most stressful situations that residents face, historically the opportunity to debrief after the death is often overlooked, scheduled only when the death was "particularly difficult," or when there was controversy by the caregivers regarding treatment decisions. ${ }^{10,11}$ In many hospital settings it appears to be considered more "art form" than "required" for team well-being, dependent on personnel and executed without consistency. The Wrap-up addressed this problem by creating an improved forum that allowed for a consistent, productive, educational, and safe environment. The survey responses have shown that this form of meeting could potentially provide meaningful support and coping skills for doctors in training when dealing with a pediatric death.

There were unique aspects of the Wrap-up that differentiate it from other forms of postdeath staff support that have been reported in the literature. ${ }^{10,11,13-16}$ Most notably, there were three key components used: (1) timeliness of the Wrapup, (2) multidisciplinary attendance, and (3) a conductor with specific skills to manage the meeting. In addition to these three components, consistency of the meetings, occurring after every death, was achieved through administrative support and commitment to the Wrap-ups from divisional leadership. 


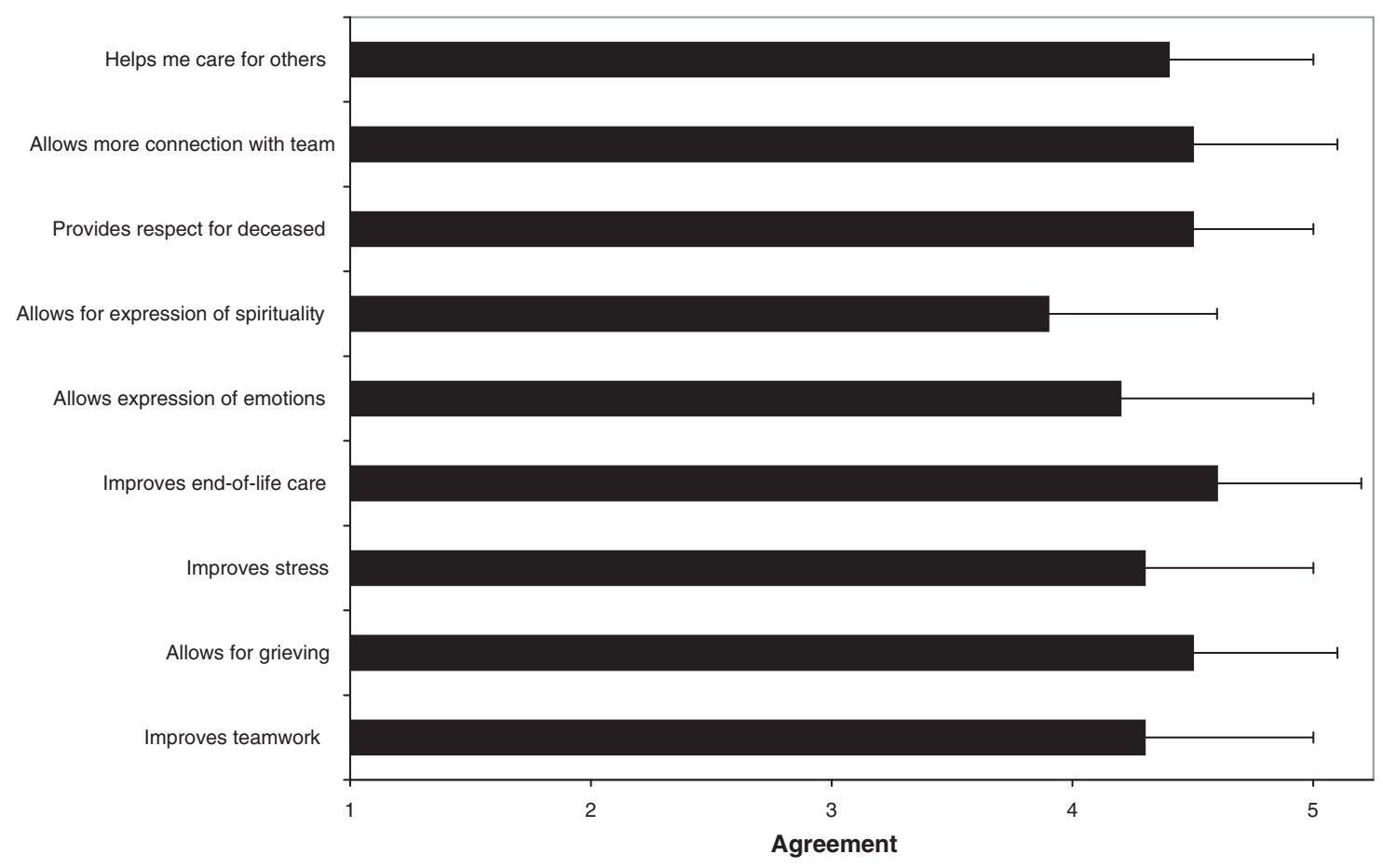

FIG. 1. Impact of Wrap-Up on pediatric residents. Average score (+SD) of resident agreement with questions posed on y axis. (Likert scale $1=$ strongly disagree, $2=$ disagree, $3=$ neutral, $4=$ agree, and $5=$ strongly agree). $\mathrm{N}=27$

Our objective was to conduct Wrap-ups within 48 hours of each death in order to assist residents and staff with real-time emotional processing of pediatric deaths. The grief reaction for the death of a pediatric patient is often dismissed and/or unprocessed as residents must return to their other sick patients and new acute situations. However, bereavement and grief can be important causes of stress and anxiety when they are not addressed. ${ }^{17}$ Therefore, attempting to address this grief early hopefully allowed for processing to alleviate anxiety and avoid denial. Personal growth and increased meaning of life have been reported to result from healthy processing of pediatric death. ${ }^{18}$ Residents surveyed in our study confirmed that the timeliness of the Wrap-up was important.

The Wrap-up has the capacity to allow the participants a variety of "lenses" as viewed by interdisciplinary team members in the collective care of the patient. Not feeling alone in one's grief is a very important aspect of grief management and a goal of grief counseling. ${ }^{12,19}$ Therefore, connecting with others on the care team through this forum may have helped their individual processing of the emotional burdens after the death. A shared experience, particularly where strong emotions are normative, can build connections within the team that cared for this patient. One of the highest ranked areas of agreement by the residents was related to the team aspect of care in the PICU, particularly with improved end-of-life care. The multidisciplinary aspect of the Wrap-up seems to foster cross-discipline awareness and empathy for others. This builds a sense of caring for each other and thus improves teamwork. The better a PICU team functions together, the better its members will be able to provide high-quality care to patients. Parents in the PICU with dying children report that trust in the PICU team, which is fostered by better teamwork, has a major impact on their experience. ${ }^{20-22}$
The conductor had an important role in the content and flow of Wrap-ups. His/her role in facilitating the meeting was key to making it a safe environment and one that fostered sharing but didn't fall into a proverbial "well of sorrow" by keeping the tenor of the sharing on a cognitive level. By this unique technique, the participants were subtly offered permission to "dip" into the affective domain knowing the conductor would bring the tenor back to a more familiar cognitive level. Accessing and expressing emotions connected to the care and death of their patient is not modeled typically. The conductor ameliorates the threat of "breaking down" or losing it by reframing the tone to a more familiar cognitive level. ${ }^{23}$ Language used by the conductor of the Wrap-up was specifically chosen to encourage broad participation and to acknowledge themes and emotions. The 30-hour formal training for conducting the Wrap-ups is requisite for those who would want to implement the Wrap-up in their hospital; in fact, the model has been replicated and implemented in a number of PICU, neonatal intensive care unit (NICU), and emergency department (ED) settings throughout the United States.

This descriptive study's evaluation is limited because of its retrospective feedback which required the residents to recollect past memories. Real-time feedback could potentially have yielded different results. A validated survey instrument would have produced results that could have allowed for statistical interpretation of the benefits of the Wrap-up. This survey was done only to elicit some formal feedback and would not have been powered to reach any statistical significance due to the small number of responders. Although the meeting was multidisciplinary, the present feedback focuses only on the physicians in training. This was done to garner the educational value of these meetings in the context of their 
learning objectives in the PICU. Clearly there are other professionals, particularly nurses, who spend a great deal of time at the bedside and are in need of support. ${ }^{24,25}$ More work on gathering the feedback of the other disciplines is needed. There are no official attendance records to determine what factors led to better attendance or what combination of staff led to a better discussion. It was clear that there were higherimpact deaths, but conducting a Wrap-up for each and every death allowed for the Wrap-up model to become part of the culture and became an expectation after a death.

\section{Conclusions}

This study shows that residents at our institution potentially benefited a great deal from participating in Wrap-ups as described in this paper. This unique forum provided a timely and facilitated discussion that positively impacted teamwork, physician stress, end-of-life care, and ultimately the ability to help others. It can be replicated at other institutions with requisite training and a commitment to creating this unique forum.

\section{Author Disclosure Statement}

The authors do not have any potential conflicts of interest to disclose.

\section{References}

1. Redinbaugh EM, Sullivan AM, Block SD, Gadmer NM, Lakoma M, Mitchell AM, Seltzer D, Wolford J, Arnold RM: Doctors' emotional reactions to recent death of a patient: Cross sectional study of hospital doctors. BMJ 2003; 327(7408):185.

2. Watson RS, Linde-Zwirbe WT, Hartman ME, et al.: ICU use at the end-of-life in US children: Robert Wood Johnson Foundation End-of-Life Peer Group. Crit Care Med 2002; 30(12S):A147.

3. Feudtner C, Christakis DA, Zimmerman FJ, et al.: Characteristics of deaths occurring in children's hospitals: Implications for supportive care services. Pediatrics 2002;109:887-893.

4. Lee KJ, Dupree CY: Staff experiences with end-of-life care in the pediatric intensive care unit. J Palliat Med 2008(9);11:986.

5. McCabe ME, Hunt EA, Serwint JR: Pediatric residents' clinical and educational experiences with end-of-life care. Pediatrics 2008;121(4):e731-e737.

6. Yang CP, Leung J, Hunt EA, Serwint J, Norvell M, Keene EA, et al.: Pediatric residents do not feel prepared for the most unsettling situations they face in the pediatric intensive care unit. J Palliat Med 2011;14(1):25-30.

7. Sack WH, Fritz G, Krener PG, Sprunger L: Death and the pediatric house officer revisited. Pediatrics 1984;73(5):676-681.

8. Truog RD, Meyer EC, Burns JP: Toward interventions to improve end-of-life care in the pediatric intensive care unit. Crit Care Med 2006;34(11 Suppl):S373-S379.

9. Baverstock A, Finlay F: Specialist registrars' emotional responses to a patient's death. Arch Dis Child 2006;91(9):774-776.
10. Serwin JL: One method of coping: Resident debriefing after the death of a patient. I Pediatr 2004;145(2):229-234.

11. Keene EA, Hutton N, Hall B, Rushton C: Bereavement debriefing sessions: An intervention to support health care professionals in managing their grief after the death of a patient. Paediatr Nurs 2010;36(4):185-189.

12. Trozzi M, Massimini K: Talking With Children About Loss. New York: Putnam-Penguin, 1999.

13. Carter BS, Guthrie SO: Utility of morbidity and mortality conference in end-of-life education in the neonatal intensive care unit. J Palliat Med 2007;10(2):375-380.

14. Dixon D, Vodde R, Freeman M, Higdon T, Mathiesen SG: Mechanisms of support: Coping with loss in a major children's hospital. Soc Work Health Care 2005;41(1):73-89.

15. Oliver RC, Sturtevant JP, Scheetz JP, Fallat ME: Beneficial effects of a hospital bereavement intervention program after traumatic childhood death. I Trauma 2001;50(3):440-448.

16. McCoyd JL, Walter C: A different kind of holding environment: A case study of group work with pediatric staff. $\mathrm{J}$ Soc Work End Life Palliat Care 2007;3(3):5-22.

17. Shear MK, Skritskaya NA: Bereavement and anxiety. Curr Psychiatry Rep 2012;14(3):169-175.

18. Taubman-Ben-Ari O, Weintroub A: Meaning in life and personal growth among pediatric physicians and nurses. Death Stud 2008;32(7):621-645.

19. Worden JW: Grief Counseling and Grief Therapy: A Handbook for the Medical Health Practioner. New York: Springer, 1991.

20. Robinson MR, Thiel MM, Backus MM, Meyer EC: Matters of spirituality at the end of life in the pediatric intensive care unit. Pediatrics 2006;118(3):e719-e729.

21. Tagarro Garcia A, Ruza Tarrio F. Parental perception of PICU end-of-life care and areas of improvement. An Pediatr (Barc) 2008;69(2):119-123.

22. Macdonald ME, Liben S, Carnevale FA, Rennick JE, Wolf SL, Meloche D, et al.: Parental perspectives on hospital staff members' acts of kindness and commemoration after a child's death. Pediatrics 2005;116(4):884-890.

23. Keene E, Hutton N, Hall B, Rushton C: Bereavement debriefing sessions: An intervention to support health care professionals in managing their grief after the death of a patient. Pediatr Nurs 2010;36(4):187.

24. Rashotte J, Fothergill-Bourbonnais F, Chamberlain M: Pediatric intensive care nurses and their grief experiences: A phenomenological study. Heart Lung 1997;26(5):372-386.

25. Meadors P, Lamson A: Compassion fatigue and secondary traumatization: Provider self care on intensive care units for children. J Pediatr Health Care 2008;22(1):24-34.

Address correspondence to: Scot T. Bateman, M.D. University of Massachusetts Medical School Department of Pediatrics, H5-524 55 Lake Avenue, North Worcester, MA 01655

E-mail: scot.bateman@umassmemorial.org 


\section{Appendix. The Anatomy of the Wrap-up}

Time:

One hour, within 48 hours of the pediatric death

\section{Participants:}

Every person who treated the patient, especially the primary caregivers of all disciplines

Norms:

The conductor will review norms at the outset:

Describe a "shared vision" for the session

Come on time, finish on time

Participate in one's own way; no one forced to talk

Only one person talks at a time: no sidebar conversations

Speak from an "I" perspective; don't assume any experiences or feelings of any other person in the room

Tolerate any expression of pain with respect: no competitive grief

Physical space:

Participants facing each other around a table
Tissues placed in the middle of the table

Narrative telling:

Conductor leads by asking someone to begin the narrative as a chronology

Then conductor lets story unfold from each participant's perspective

Listens for themes (failure, gratitude, guilt, cooperation, etc.) and acknowledges them

Regulates the emotive temperature of the group

Regulates participation, allowing all to speak

Reflects and acknowledges information as it unfolds as well as feelings

Remains neutral and compassionate for the participants

Drives the pace by remaining soothing and slow

\section{Closing:}

Reacknowledges themes

Discusses the value of reflection in the context of ongoing work 